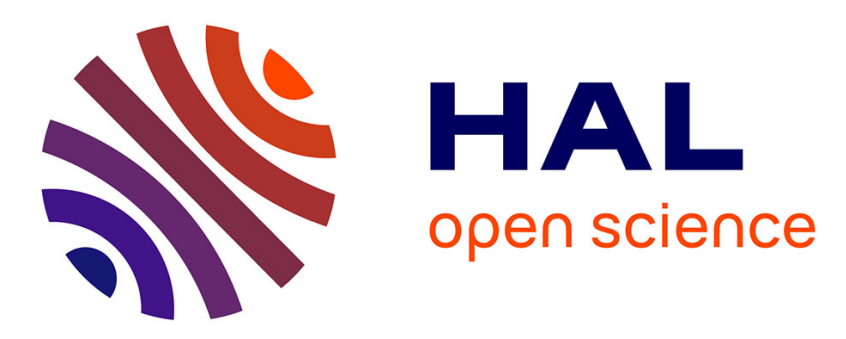

\title{
Biomechanical interests of supra-cervical hysterectomy with sacrocolpopexy: first study using finite element modeling
}

Marine Lallemant, Abel Vega, Jérôme Chambert, Emmanuelle Jacquet, Rajev

Ramanah

\section{To cite this version:}

Marine Lallemant, Abel Vega, Jérôme Chambert, Emmanuelle Jacquet, Rajev Ramanah. Biomechanical interests of supra-cervical hysterectomy with sacrocolpopexy: first study using finite element modeling. International Urogynecology Journal, 2021, 32, pp.1599 - 1502. hal-03549371

\section{HAL Id: hal-03549371 \\ https://hal.science/hal-03549371}

Submitted on 31 Jan 2022

HAL is a multi-disciplinary open access archive for the deposit and dissemination of scientific research documents, whether they are published or not. The documents may come from teaching and research institutions in France or abroad, or from public or private research centers.
L'archive ouverte pluridisciplinaire HAL, est destinée au dépôt et à la diffusion de documents scientifiques de niveau recherche, publiés ou non, émanant des établissements d'enseignement et de recherche français ou étrangers, des laboratoires publics ou privés. 


\title{
Biomechanical interests of supra-cervical hysterectomy with sacrocolpopexy: first study using finite element modeling
}

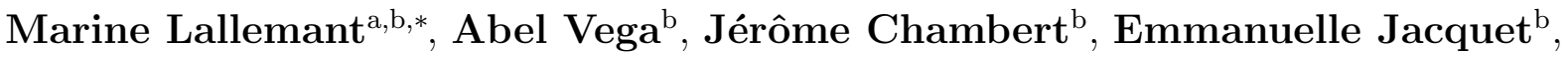 \\ Rajeev Ramanah ${ }^{\mathrm{a}, \mathrm{b}}$ \\ ${ }^{a}$ Department of Gynecologic Surgery, Besançon University Medical Centre, 3 Alexander Fleming \\ Boulevard, 25000 Besançon, France \\ ${ }^{b}$ FEMTO-ST Institute, Bourgogne Franche-Comté University, CNRS, Besançon, France
}

Keywords: Finite element modeling, Sacrocolpopexy, Hysterectomy, Pelvic organ prolapse, Magnetic resonance imaging

\section{Introduction}

Laparoscopic sacrocolpopexy (LSC) is a common surgery performed for pelvic organ prolapse (POP). Hysterectomy can be associated with this procedure, but its usefulness is debated (longer surgical time, more perioperative blood loss, higher risk for mesh exposure, probably less prolapse recurrence, no evidence for the sexual benefit)[1]. The finite element method (FEM), which is a numerical method for solving biomechanical and biomedical problems, has been used to study POP. Finite element simulations have been performed to compare sacrocolpopexy with and without supra-cervical hysterectomy for POP to test the hypothesis that hysterectomy could reduce the stress and strain of mechanical fields on pelvic organs and on apical supporting ligaments.

\section{Case study}

Magnetic resonance (MR) pre- and post-operative images of two patients, one having LSC with and one without supracervical hysterectomy for a POP repair, were used. 3D

\footnotetext{
*Corresponding author: Marine Lallemant, mlallemant@chu-besancon.fr
} 
models of the pelvic organs were constructed using Slicer@ (Fig. 1). The 3D model was transformed into a geometric mesh model using Rhinoceros $\mathbb{R}$ before creating a solid model using SolidWorks@ (Fig. 2). The biomechanical study was performed by using the FE method software ANSYSR with linear elastic behavior and material properties of the organs and the ligaments from the literature [2]. Pelvic organs were exposed to an intra-abdominal pressure of $0.20 \mathrm{MPa}$ to simulate a Valsalva maneuver at an inclination of $45^{\circ}$ from the sagittal axis in an antero-posterior direction. The levator plate was assumed to be a fixed support.

Prior to surgery, there was no large difference in terms of POP-Q classification of the prolapse: stage 2 for the anterior compartment for both patients, stage 2 for the apical compartment for both patients, and stage 2 and stage 1 for the posterior compartment of each patient. The volume of the uterus was equivalent $\left(110,000 \mathrm{~mm}^{3}\right.$ and $\left.90,000 \mathrm{~mm}^{3}\right)$.

Subsequently, the equivalent stress (or the equivalent strain) is a scalar value that represents the Cauchy stress field (or the strain field). FE modeling simulation showed that equivalent stress increased on pelvic organs after LSC both with (4.92 MPa before versus 6.81 MPa after surgery) and without supracervical hysterectomy (8.84 MPa before versus 30.34 MPa after surgery; Fig. 3). However, equivalent strain on pelvic organs decreased after LSC with supracervical hysterectomy ( 0.12 before versus 0.08 after surgery), but increased in the case of uterine preservation ( 0.19 before versus 0.67 after surgery; Fig. 4). Stress and strain on the left and right cardinal ligaments decreased after both types of surgery. An asymmetrical distribution of stress and strainwas observed on apical supporting ligaments with a post-operative increase on the left uterosacral ligament following LSC with and without supra-cervical hysterectomy (Fig. 5) because the mesh is placed on the right.

In our study, LSC without a supracervical hysterectomy was associated with a higher strain on pelvic organs. An asymmetrical distribution of stress and strain was observed. These biomechanical results could explain some cases of prolapse recurrence and pain after LSC. In the future, a new study could be carried out that would include more patients and a statistical comparison of them. 

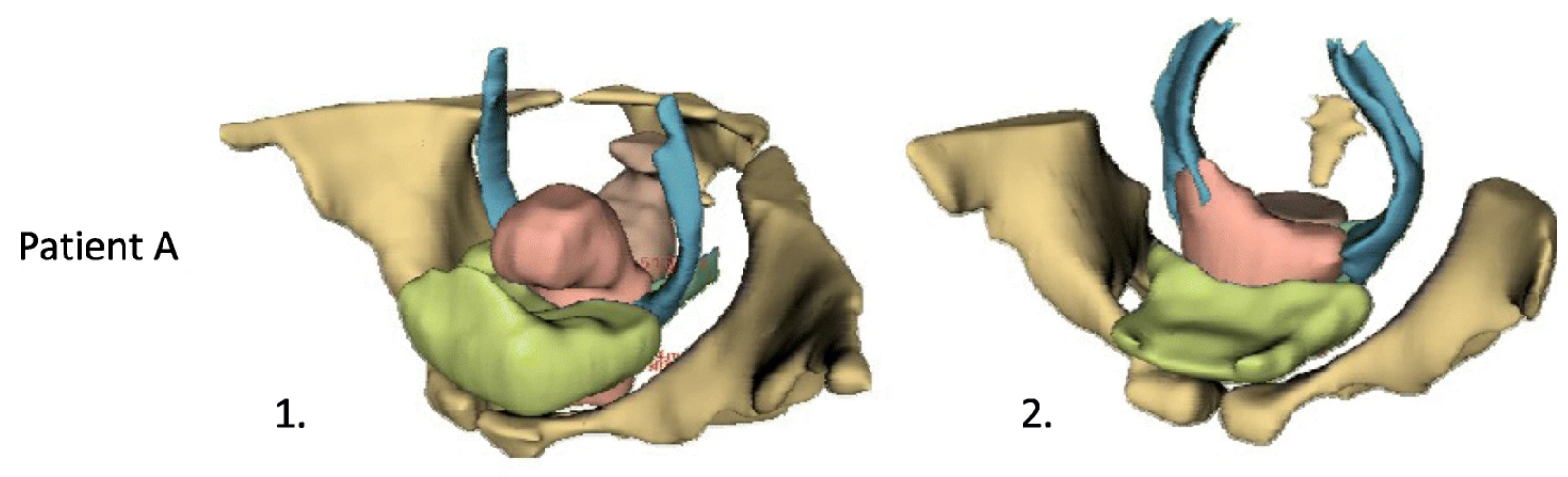

\section{Patient B}
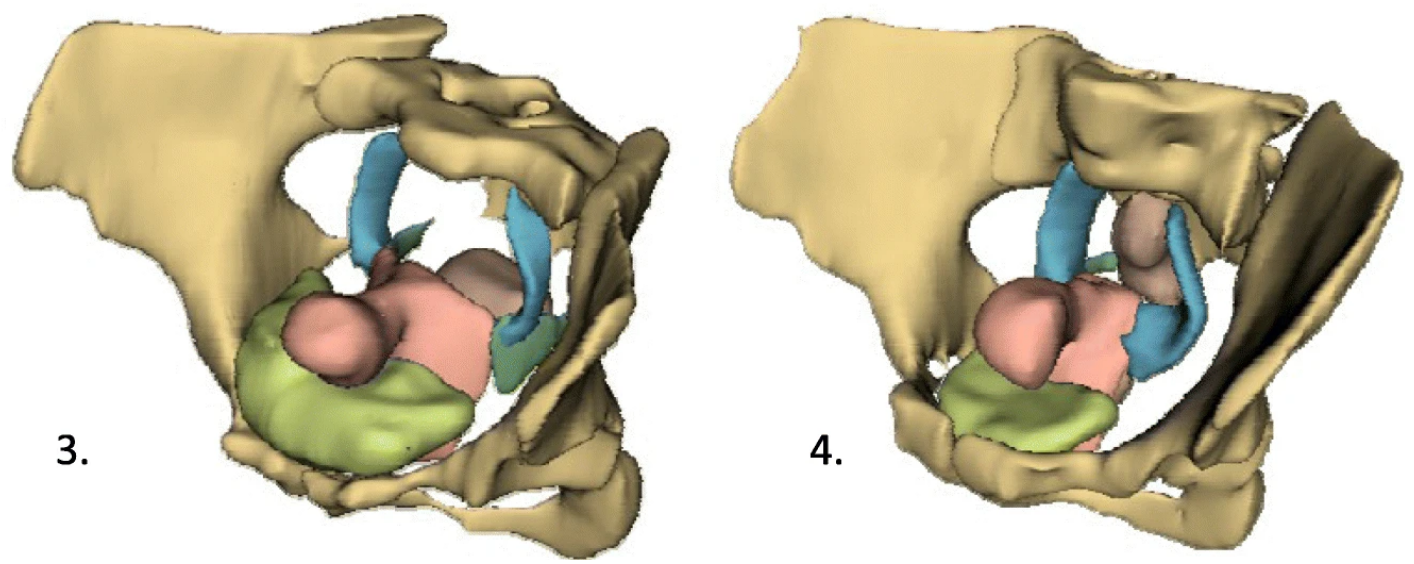

Figure 1: 3D models of pelvic organs, cardinal and uterosacral ligaments. Patient A Laparoscopic sacrocolpopexy (LSC) with a supra-cervical hysterectomy. Patient B LSC without supra-cervical hysterectomy. 1 Before LSC with supra-cervical hysterectomy. 2 After LSC with supra-cervical hysterectomy. 3 Before LSC without supracervical hysterectomy. 4 After LSC without supra-cervical hysterectomy. Bladder is shown in light green, uterus and vagina are shown in pink, the rectum is shown in gray, the cardinal ligaments are shown in blue, the uterosacral ligaments are shown in dark green, and the pelvic bone is shown in yellow 

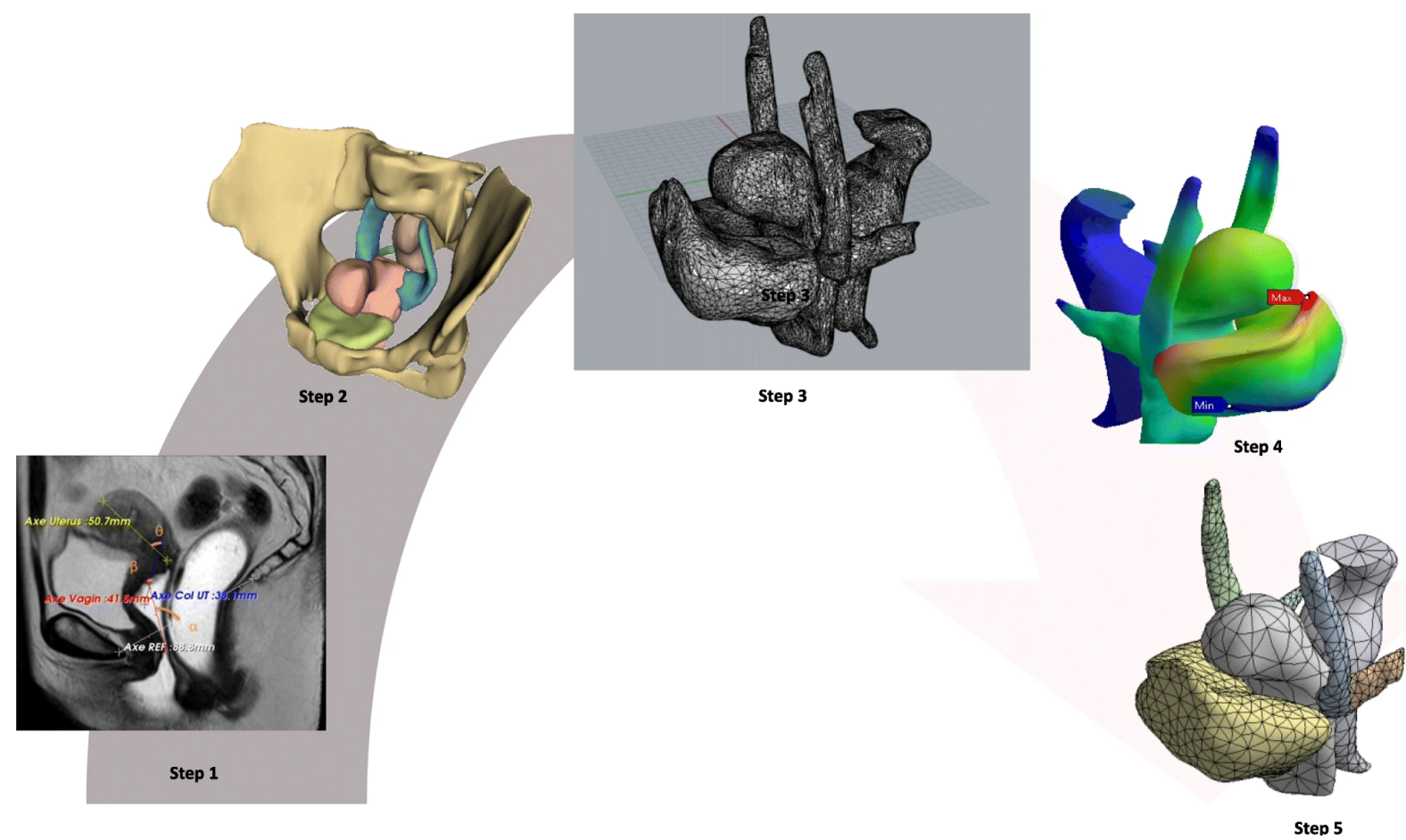

Figure 2: Steps to obtaining a finite element (FE) analysis model of the pelvic organs. An FE model is a combination of mathematical modeling and a computer simulation for studying organ mobilities. Step 1 Preand post-operative MRI. Step 2 Construction of a 3D model of the pelvic organs. Step 3 Transformation of the 3D mode into a geometric mesh model. Step 4 Creation of a solid model. Step 5 Numerical simulation: performing a biomechanical study using the FE method after importing the solid model into ANSYS® software and using linear elastic mechanical characteristics from the literature 


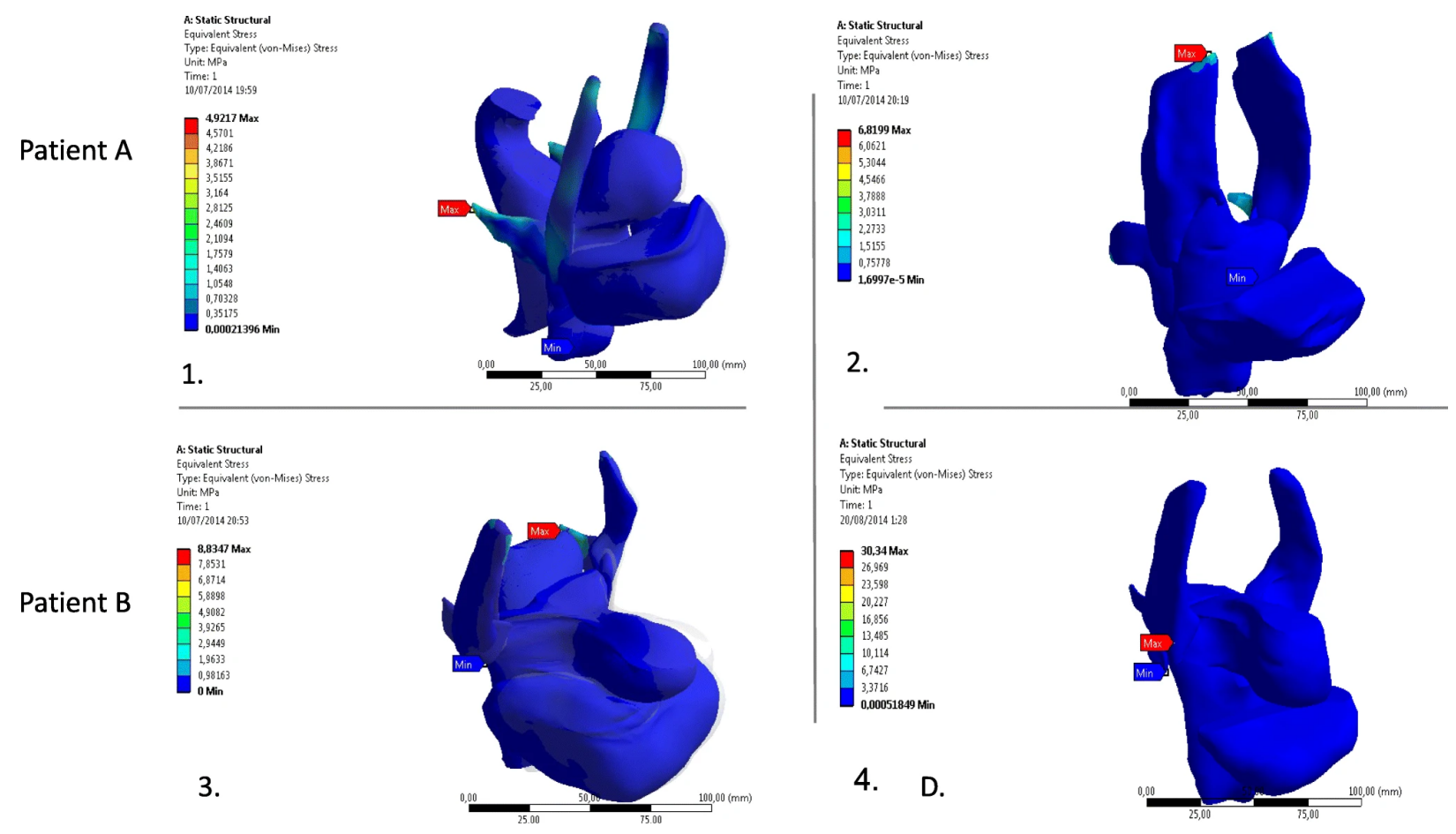

Figure 3: Equivalent stress on pelvic organs shown by finite element (FE) modeling simulation after laparoscopic sacrocolpopexy (LSC). Patient A LSC with supra-cervical hysterectomy. Patient B LSC without supracervical hysterectomy. 1 Before LSC with supra-cervical hysterectomy. 2 After LSC with supracervical hysterectomy. 3 Before LSC without supra-cervical hysterectomy. 4 After LSC without supracervical hysterectomy. The bottom scale is a length scale $(\mathrm{mm})$ 


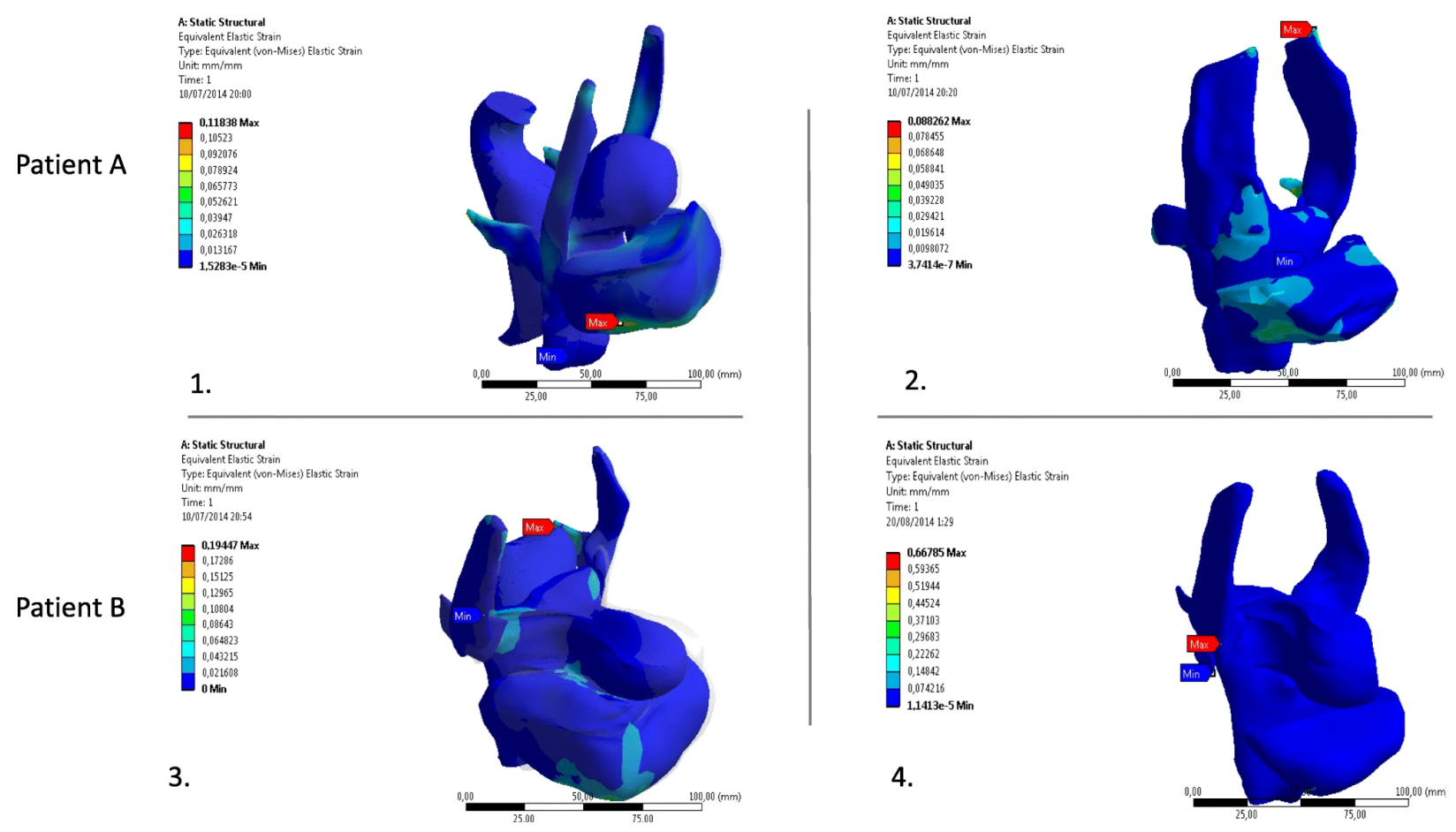

Figure 4: Equivalent elastic strain on pelvic organs studied by finite element(FE) modeling simulation before and after laparoscopic sacrocolpopexy (LSC). Patient A LSC with supra-cervical hysterectomy. Patient $B$ LSC without supra-cervical hysterectomy. 1 Before LSC with supra-cervical hysterectomy. 2 After LSC with supra-cervical hysterectomy. 3 Before LSC without supra-cervical hysterectomy. 4 After LSC without supracervical hysterectomy. The bottom scale is a length scale $(\mathrm{mm})$ 


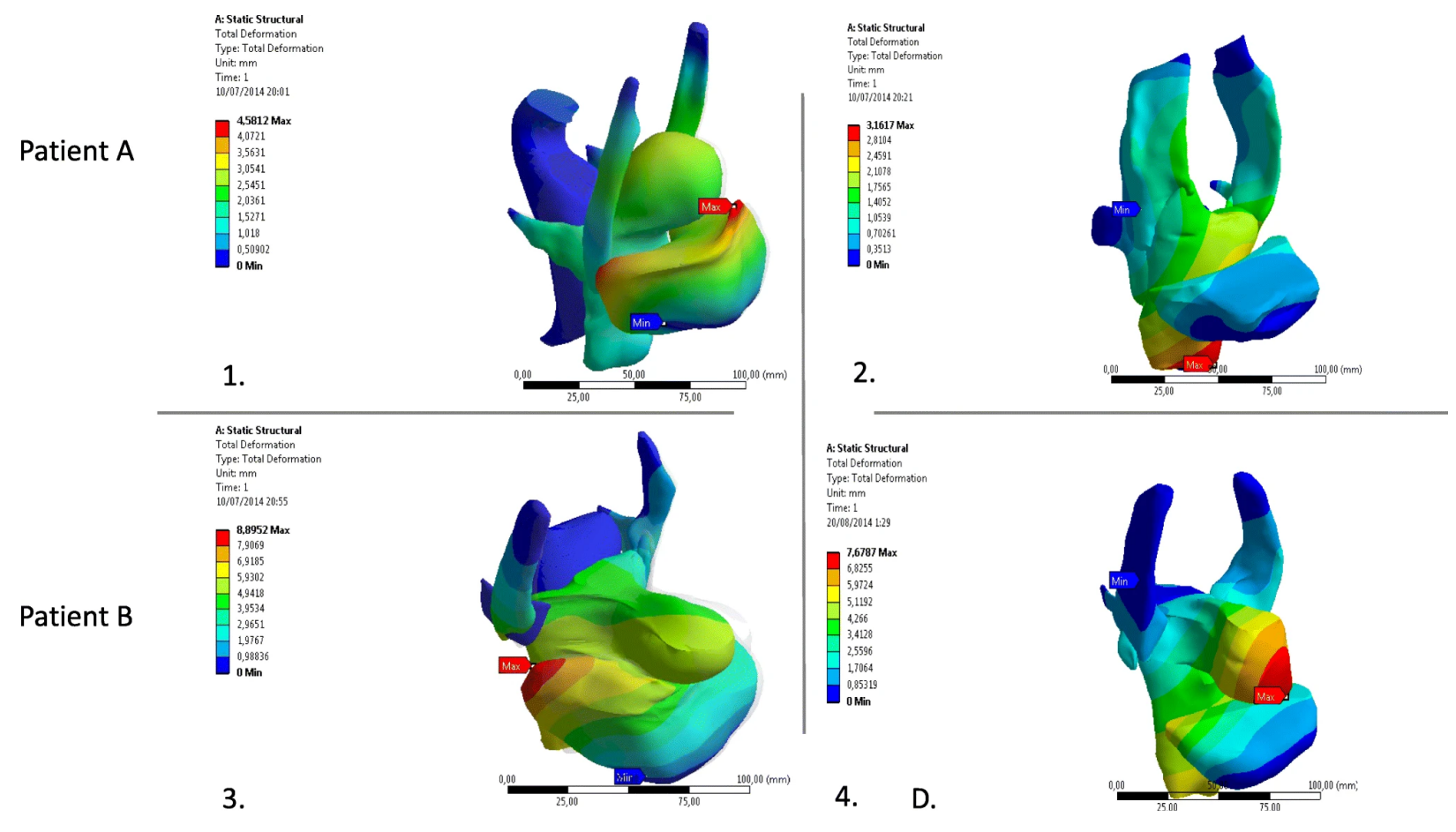

Figure 5: Total deformation of pelvic organs studied by finite element (FE) modeling simulation before and after laparoscopic sacrocolpopexy (LSC). The total deformation is defined by the deflection of organs after applying not only fixed support on the levator plate but also intra-abdominal pressure. These figures show how the organs are deformed by also taking into account mechanical contact between the organs. Patient A LSC with supra-cervical hysterectomy. Patient B LSC without supra-cervical hysterectomy. 1 Before LSC with supra-cervical hysterectomy. 2 After LSC with supra-cervical hysterectomy. 3 Before LSC without supra-cervical hysterectomy. 4 After LSC without supra-cervical hysterectomy. The bottom scale is a length scale $(\mathrm{mm})$ 
Contributions M. Lallemant: data analysis, manuscript writing/editing; A. Vega: protocol/project development, data collection or management, data analysis; J. Chambert: protocol/project development, data collection or management, data analysis, manuscript writing/editing; E. Jacquet: protocol/project development, data collection or management; data analysis, manuscript writing/editing; R. Ramanah: protocol/project development, data collection or management, data analysis, manuscript writing/editing.

\section{Declarations}

Conflicts of interest None.

Consent Written informed consent was obtained from the patients for publication of this Images in Urogynecology and any accompanying images.

\section{References}

\section{References}

[1] Cayrac M, Warembourg S, Le Normand L, Fatton B. [Does hysterectomy modifies the anatomical and functional outcomes of prolapse surgery?: Clinical practice guidelines]. Prog Urol 2016;26(Suppl 1):S7388.

[2] Rubod C, Brieu M, Cosson M, Rivaux G, Clay JC, de Landsheere L, et al. Biomechanical properties of human pelvic organs. Urology 2012;79(4):968.e17-22. 